\title{
NATURAL CONVECTION IN A PARTIALLY HEATED CYLINDER: A NUMERICAL STUDY
}

\author{
José NÚÑEZ \\ Escuela Nacional de Estudios Superiores, Unidad Morelia, Universidad Nacional Autónoma de México, \\ Morelia, Michoacán, México \\ e-mail: joseng@enesmorelia.unam.mx \\ Alberto Beltrán, Michel Rivero \\ Instituto de Investigaciones en Materiales, Unidad Morelia, Universidad Nacional Autónoma de México, \\ Morelia, Michoacán, México
}

\begin{abstract}
This work presents a numerical study on a natural convective flow in a cylindrical container heated from below, cooled from above, and partially heated from the lateral wall. Mass, momentum and energy equations were solved with a developed hybrid Fourier-finite volume code and validated with the commercial software COMSOL Multiphysics for steady-state solutions. The primary solutions correspond to steady-states $C_{m}$ with azimuthal wavenumbers $m$. The results show mode competition between different states leading to many flow solutions including steady axisymmetric, steady non-axisymmetric, time-dependent pulsating wave solutions, and other flow states with a variety of spatiotemporal symmetries.
\end{abstract}

Keywords: bifurcations, natural convection, partially heated cylinder, switching modes solutions

\section{Introduction}

Thermal instabilities in natural convection flows are a subject of great interest due to the role that they play in many technological applications like crystal growth, processing of molten metals, and liquid metal batteries (LMBs). For instance, Lappa (2005) reported instabilities of liquid metal layers to model crystal growth processes. Son and Yi (2005) studied sources of flow instabilities for melt in a large crucible. For energy storage devices, like LMBs, the Joule heating generates convective motion in three layers of conducting fluids (low Prandtl number) segregated by their differences in density and confined in a cylindrical vessel. In particular, this heating is mostly localized in the center volume of the vessel occupied by a thin layer of a molten salt electrolyte, which separates two metals in the liquid state. In the numerical work by Shen and Zikanov (2016), it was found that convection is always present in small laboratory prototypes, and it might become stronger in larger systems. In general, the appearance of hydrodynamic instabilities leads to flows that may affect/improve the battery operation, depending on the flow patterns. From the experimental point of view, the evidence of the positive effect of convection as a mechanism of mixing in a single layer of a liquid metal (liquid metal electrode) was reported by Kelley and Sadoway (2014), and it was confirmed in numerical simulations by Beltrán (2017). The experimental setup used by Kelley and Sadoway (2014) enforces convection by using a partially heated cylinder (heating from below and cooling the lateral and top walls).

In the literature, there has been a wide discussion about natural convection with partial heating or partial active walls. Special attention was focused on two-dimensional square cavities partially heated from below and from the sides (Hasnaoui et al., 1992). The majority of simulations have been performed for various lengths, positions and inclinations of the heated source 
(Nithyadevi et al., 2007; Ben-Cheikh et al., 2010; Al-Rashed et al., 2017; Varol et al., 2009). Analysis of how different boundary conditions influence the thermal field and the effects in the determination of the local and averaged heat transfer coefficients were also investigated (Padilla et al., 2013). Results show that the Nusselt number increases with the increasing heater size and the Rayleigh number (Cianfrini et al., 2013). Three-dimensional numerical studies reported multiple states and bifurcations on natural convection in a rectangular cavity with partially heated vertical walls (Gelfgat, 2017). The study of natural convection has been extended to nanofluids (Nascimento et al., 2019). Correlation equations for the Nusselt number in terms of the Rayleigh number, heated length, and volume fraction were reported in partially heated cylindrical enclosures (Guestal et al., 2018).

On the other hand, the phenomena of symmetry breaking and transition to unsteady non-symmetric convection for fluids with a low Prandtl number are commonly found in electromagnetic processing of materials and other applications. For instance, Bennacer et al. (2006) studied numerically the symmetry breaking of the melt flow in a Bridgman configuration. It consists of a cartesian domain filled with a liquid metal heated from below and cooled from above, and partially heated from the lateral walls. They noticed that symmetry breaking firstly occurs in the transverse plane and also that the heat transfer increases locally on the bottom and decreases on the vertical active walls. Erenburg et al. (2003) investigated multiple states, stability, and bifurcations of natural convection in a rectangular cavity with partially heated vertical walls. The appearance of complicated multiplicity is explained by development of stably and unstably stratified regions, where damping and Rayleigh-Bénard instability mechanisms compete with the primary buoyancy force localized near the heated parts of the vertical boundaries.

Most studied cases involve symmetry breaking from a steady axisymmetric state to a rotating wave (Lopez and Marques, 2009; Gutierrez-Castillo and Lopez, 2017). Besides, Núñez et al. (2018) performed a numerical study dealing with the symmetry breaking of natural convection in a cylindrical cavity heated from below and partially cooled from above. Nam and Yi (2010) performed simulation of thermal fluctuation according to the melt height in a Czochralski growth system for a cylindrical crucible. The thermal wave patterns exhibited transition behavior from a 3-pole-wave pattern to a 6 -pole-wave pattern when the melt height was decreased from 0.14 to $0.1 \mathrm{~m}$.

The present numerical study focuses on the effect of dimensionless parameters like the Rayleigh and Prandtl numbers as well as the heater size and the aspect ratio on the natural convective flow in a cylindrical container with a partially heated lateral wall. A hybrid Fourier-finite volume method is used to solve the governing equations (Núñez et al., 2012a) as well as the commercial software COMSOL Multiphysics. Temperature distributions are presented and the heat transfer rate is evaluated in terms of the Nusselt number. When the Rayleigh number is varied, the onset of non-axisymmetric natural convection is studied based on a nonlinear numerical instability analysis (Núnez et al., 2018). Apparently, and to the best of our knowledge, such a detailed study has not been previously performed.

The paper is organized as follows. In Section 2, we describe the physical model under study. In Section 3, we provide a brief description of the natural convection flow and the assumptions under which our problem will be set, including all governing equations, boundary conditions, and the numerical methodology. In Section 4, the numerical results are discussed. Finally, the concluding remarks are given in Section 5.

\section{Problem formulation}

The schematic diagram of the system under consideration is shown in Fig. 1. It consists of a cylindrical container heated from below and cooled from above, with a partially heated lateral wall. To characterize the geometrical configuration, two relevant dimensionless parameters are 
used: the heater size $\gamma=h / H$, and the aspect ratio $a=H / D$; where $h$ is the heated height on the lateral wall, $H$ and $D$ are the height and the diameter of the cylinder, respectively. The heater size is varied in the range of $0 \leqslant \gamma \leqslant 1$, where zero corresponds to the whole adiabatic lateral wall and $\gamma>0$ resembles a configuration partially heated from the side. Moderate aspect ratios, between $0.5 \leqslant a \leqslant 1.25$, are selected to maintain a basic single roll flow configuration. Cylinders out of this range, typically introduce new instabilities, which break the basic roll into smaller ones (Lappa, 2010). These geometries are out of the scope of this work. Since this work is motivated by the understanding of the underlying physics on natural convective flows commonly encountered on several applications, the Prandtl numbers ranging from 0.001 to 6.67 are studied.

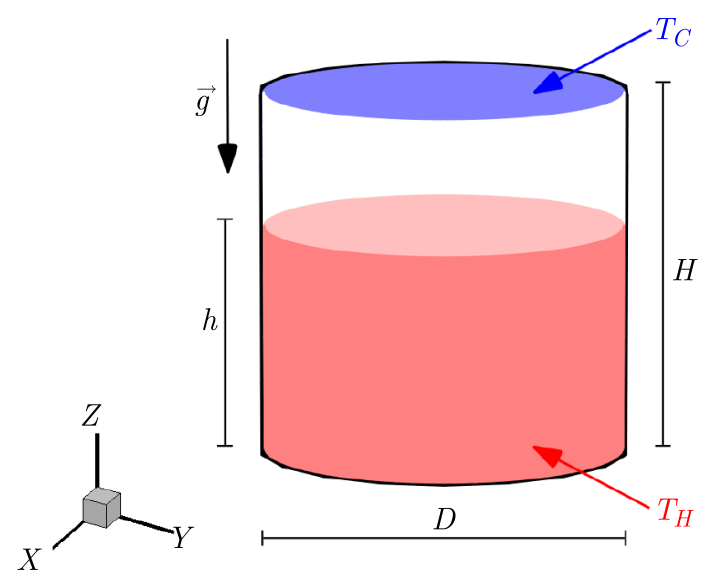

Fig. 1. Schematic diagram of the flow configuration. Red region (bottom wall and side wall up to height $h$ ) has uniform temperature $T_{H}$. Blue region (upper wall) has uniform temperature $T_{C}$.

Remaining side wall (white region) is considered adiabatic

\section{Governing equations}

The flow in the cylindrical cavity described in the previous Section is governed by the coupled Navier-Stokes (mass and momentum) and energy equations. The Boussinesq approximation is considered. The set of conservation equations have been solved in its dimensionless form. The scaling we have used was the most convenient for numerical solution (Núñez, 2012b) and included the following characteristic quantities: the axial and radial coordinates are expressed in terms of the height $H$ and diameter $D$ of the cylinder, respectively. The characteristic velocity $u_{c}$ is the free-fall velocity, namely $u_{c}=\sqrt{g \beta \Delta T h}$ where $g$ is the gravity acceleration. The symbol $\Delta T=T_{H}-T_{C}$ denotes the characteristic temperature scale with $T_{H}$ and $T_{C}$ being the temperatures of the lower and upper walls, respectively. The time scale is defined using the characteristic velocity as $h / u_{c}$. The governing equations are written in cylindrical coordinates $(r, \theta, z)$ as follows

$$
\begin{aligned}
& \frac{1}{r} \frac{\partial r u_{r}}{\partial r}+\frac{1}{r} \frac{\partial u_{\theta}}{\partial \theta}+\frac{\partial u_{z}}{\partial z}=0 \\
& \frac{\partial u_{r}}{\partial t}+(\mathbf{u} \cdot \nabla) u_{r}-\frac{u_{\theta}^{2}}{r}=-\frac{\partial p}{\partial r}+\sqrt{\frac{\operatorname{Pr}}{\operatorname{Ra}}}\left(\nabla^{2} u_{r}-\frac{u_{r}}{r^{2}}-\frac{2}{r^{2}} \frac{\partial u_{\theta}}{\partial \theta}\right) \\
& \frac{\partial u_{\theta}}{\partial t}+(\mathbf{u} \cdot \nabla) u_{\theta}+\frac{u_{\theta} u_{r}}{r}=-\frac{1}{r} \frac{\partial p}{\partial \theta}+\sqrt{\frac{\operatorname{Pr}}{\operatorname{Ra}}}\left(\nabla^{2} u_{\theta}-\frac{u_{\theta}}{r^{2}}+\frac{2}{r^{2}} \frac{\partial u_{r}}{\partial \theta}\right) \\
& \frac{\partial u_{z}}{\partial t}+(\mathbf{u} \cdot \nabla) u_{z}=-\frac{\partial p}{\partial z}+\sqrt{\frac{\operatorname{Pr}}{\operatorname{Ra}}} \nabla^{2} u_{z}+T \\
& \frac{\partial T}{\partial t}+(\mathbf{u} \cdot \nabla) T=\sqrt{\frac{1}{\operatorname{Ra} \operatorname{Pr}}} \nabla^{2} T
\end{aligned}
$$


The operators are defined as

$$
\mathbf{u} \cdot \nabla=u_{r} \frac{\partial}{\partial r}+\frac{u_{\theta}}{r} \frac{\partial}{\partial \theta}+u_{z} \frac{\partial}{\partial z}
$$

and

$$
\nabla^{2}=\frac{1}{r} \frac{\partial}{\partial r}\left(r \frac{\partial}{\partial r}\right)+\frac{1}{r^{2}} \frac{\partial^{2}}{\partial \theta^{2}}+\frac{\partial^{2}}{\partial z^{2}}
$$

where $\mathbf{u}=\left[u_{r}, u_{\theta}, u_{z}\right]$ is velocity, $p$ is pressure, and $T$ is temperature. $\operatorname{Pr}=\nu / \alpha$ is the Prandtl number, and $\mathrm{Ra}=g \beta\left(T_{H}-T_{C}\right) h^{3} /(\nu \alpha)$ is the Rayleigh number.

For the given configuration, the upper wall has a uniform temperature $T_{C}$. The bottom wall and side wall up to the height $h$ have a uniform temperature $T_{H}$. The remaining side wall is considered adiabatic. Therefore, thermal boundary conditions at the bottom (hot) and top (cold) walls are given by

$$
T=\left\{\begin{array}{lll}
1 & \text { at } & z=0 \\
0 & \text { at } & z=1
\end{array}\right.
$$

The thermal boundary condition for the lateral wall plays a major role in the flow. For the configuration with partial heating from the side, the following boundary conditions are considered

$$
T=1 \quad \text { for } \quad 0 \leqslant z<\gamma
$$

and

$$
\frac{\partial T}{\partial r}=0 \quad \text { for } \quad \gamma \leqslant z \leqslant 1
$$

For the velocity, the no-slip boundary condition is used

$$
\mathbf{u}=0 \quad \text { on all walls }
$$

The initial conditions are

$$
\mathbf{u}=0 \quad \text { and } \quad T=0.5 \text { for } t=0
$$

To evaluate the effect of convective flow on the heat transfer of our problem, the mean Nusselt number is evaluated on the top surface $S$. It is defined as

$$
\mathrm{Nu}=-\left.\frac{1}{S} \int_{S} \frac{\partial T}{\partial z}\right|_{z=1} d S
$$

To quantify the relative influence of, and switching between, different modes, the kinetic energy of the azimuthal Fourier modes is used. It is defined as

$$
K E_{m}=\frac{1}{2} \int_{z=0}^{z=1} \int_{r=0}^{r=r_{e}} \mathbf{u}_{m} \cdot \mathbf{u}_{m}^{*} r d r d z
$$

where $\mathbf{u}_{m}$ is the Fourier mode of the velocity corresponding to the wavenumber $m$, and $\mathbf{u}_{m}^{*}$ is its complex conjugate.

The numerical procedure implemented to solve Eqs. (3.1), subjected to the boundary conditions given by Eqs. (3.5)-(3.7), is described in the next Section. 


\subsection{Numerical methodology}

A numerical solution is obtained for the velocity and temperature fields with a hybrid Fourier-finite volume code (Núnez, 2012a). The Fourier method has the advantage of a high-order approximation for the partial derivatives in the angular direction and it guarantees the azimuthal periodic boundary condition. By using the finite volume method in the $r$ and $z$ directions, discontinuous boundary conditions in those directions can be handled. The convective and diffusive terms are calculated with a central difference scheme. The Euler method is used for the time integration. The SIMPLEC algorithm (Patankar and Spalding, 1972) is applied for correction of the velocity components and pressure. This algorithm is iterative and stops with a small velocity divergence criterion, $\epsilon=10^{-5}$. In general, this methodology is well suited for stability and symmetry breaking analysis of viscous flows.

Given the lack of experimental measurements for the configuration addressed in this work, the hybrid Fourier-finite volume code was validated with PIV experimental results reported by Ramírez et al. (2020) for a cylindrical vessel heated from below and cooled from above. The working fluid was water. This validation was done for the case with no lateral partial heating, by fixing $\gamma=0$. In Fig. 2a and 2b, experimental and numerical velocity fields for the $r-z$ plane are presented, respectively. In general, a single recirculation can be distinguished. Figure $2 \mathrm{c}$ shows a comparison between experimental and numerical results for the velocity components $(u, v, w)$ along the diameter and for $z=0.5$, where the higher velocity is expected. Continuous lines and markers correspond to the numerical solution and experimental measurements, respectively. It should be noted that these results are in good agreement when considering the corresponding experimental uncertainty.

In addition to our code development, the COMSOL Multiphysics 5.5 software is also used in the present study to look for steady-state solutions. This commercial software was also used to model the experimental results previously presented, and a similar comparison (not shown) was obtained.

(a)

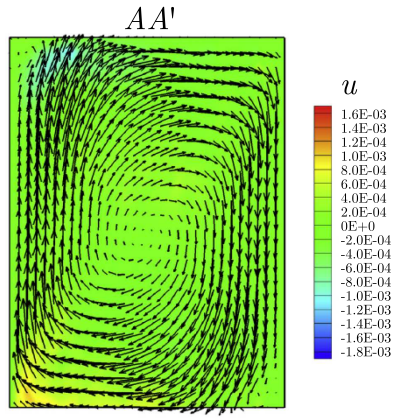

(b)

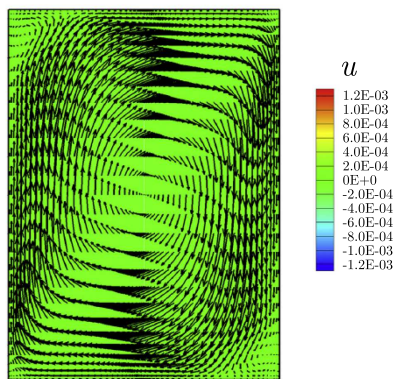

(c)
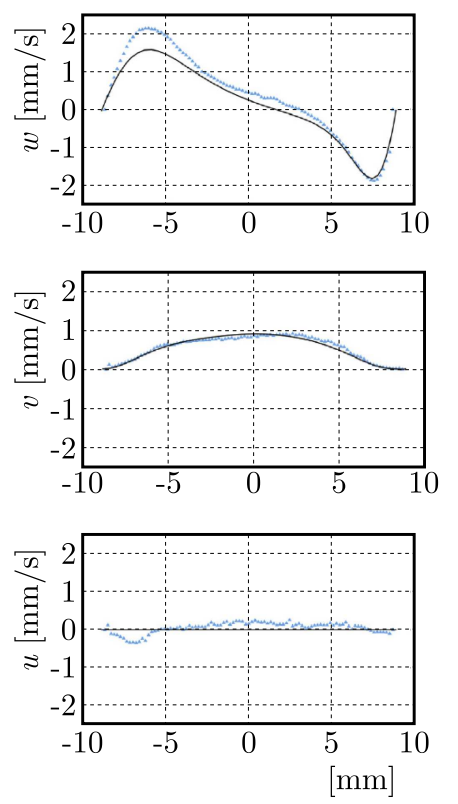

Fig. 2. Velocity fields for $\theta=0$ : (a) experimental measurements, (b) numerical results, (c) velocity components $(u, v, w)$ along the diameter and for $z=0.5$. Continuous lines and markers correspond to the numerical solution and experimental measurements, respectively 
For the considered flow (steady-state laminar flow under specified conditions), several parameters have been tested and variables logged to make a grid sensitivity study. In Table 1, four different grids were explored, and the main results are presented for the $\mathrm{Nu}$ number and the maximum axial velocity $w_{\max }$ with $\gamma=0.5, a=1$, and $\mathrm{Ra}=10^{4}$. In all cases, the dimensionless variables exhibited low influence on the grid size.

Table 1. Grid sensitivity study for the Nusselt number and the maximum axial velocity

\begin{tabular}{|c|c|c|}
\hline$n_{\theta} \times n_{r} \times n_{z}$ & $\mathrm{Nu}$ & $w_{\max }$ \\
\hline \hline $30 \times 30 \times 30$ & 2.542 & $6.431 \cdot 10^{-2}$ \\
\hline $30 \times 40 \times 40$ & 2.534 & $6.390 \cdot 10^{-2}$ \\
\hline $42 \times 60 \times 60$ & 2.528 & $6.356 \cdot 10^{-2}$ \\
\hline $42 \times 60 \times 80$ & 2.527 & $6.342 \cdot 10^{-2}$ \\
\hline
\end{tabular}

The results given in Table 1 show that a grid size of $42 \times 60 \times 60$ satisfies the grid independence. Under similar conditions in the COMSOL software, a Nu number of 2.522 was obtained, which represents a difference of $0.2 \%$ when compared to our code. The comparison of all results allows cross-validation of both models.

\section{Results and discussion}

The numerical solution for the heat transfer and the fluid flow in a system where the equations and boundary conditions are invariant to arbitrary azimuthal rotations must preserve the rotation invariance. For the numerical solution, at a moderate Ra number $\mathrm{Ra}<10^{3}$, the motion consists of a steady axisymmetric flow; whereas, on increasing Ra, the flow undergoes throughout a series of bifurcations. When a symmetry-breaking bifurcation occurs in the flow, new states appear, and they have less symmetry and often more complicated dynamics (Crawford, 1991). In the next Sections, we present the basic states and bifurcation analysis of the flow.

\subsection{Basic states}

Lateral partial heating introduces a new instability process in natural convection flows. To get an insight, a comprehensive and systematic study is implemented to analyze the relative importance of the dimensionless parameters. To show the results for different Fourier modes solutions, the temperature field is reported at two mutually perpendicular planes for most of the considered cases. The velocity field, which can be inferred from the temperature field, is not presented to keep short the results presentation. In the following subsections, the effect of different parameters is discussed independently.

\subsubsection{Heater size}

In this Section, we explore the effect of the heater size $\gamma$ on the flow while maintaining the other parameters constant, namely, $\mathrm{Ra}=10^{4}, \operatorname{Pr}=6.67$ and $a=1.0$. Figure 3 shows the temperature distributions and isotherms for $\gamma=0,0.25,0.5,0.75$, and 1.0, from the left to right. The upper row is the plane at the angular orientation, $\theta=0$, and the lower row is a perpendicular plane at $\theta=\pi / 2$. For $\gamma=0,0.25$, and 0.5 , non-axisymmetric solutions $C_{1}$ were found; however, the behaviour changes when the heater size increases. For, $\gamma=0.75$ and 1.0, non-axisymmetric solutions become axisymmetric ones $C_{0}$. It is observed that there is a smooth transition from $C_{1}$ to $C_{0}$. The symmetry breaking bifurcation occurs for the critical heater size value of $\gamma_{c}=0.727$, obtained from the nonlinear stability analysis (Núñez and Beltrán, 2018). 

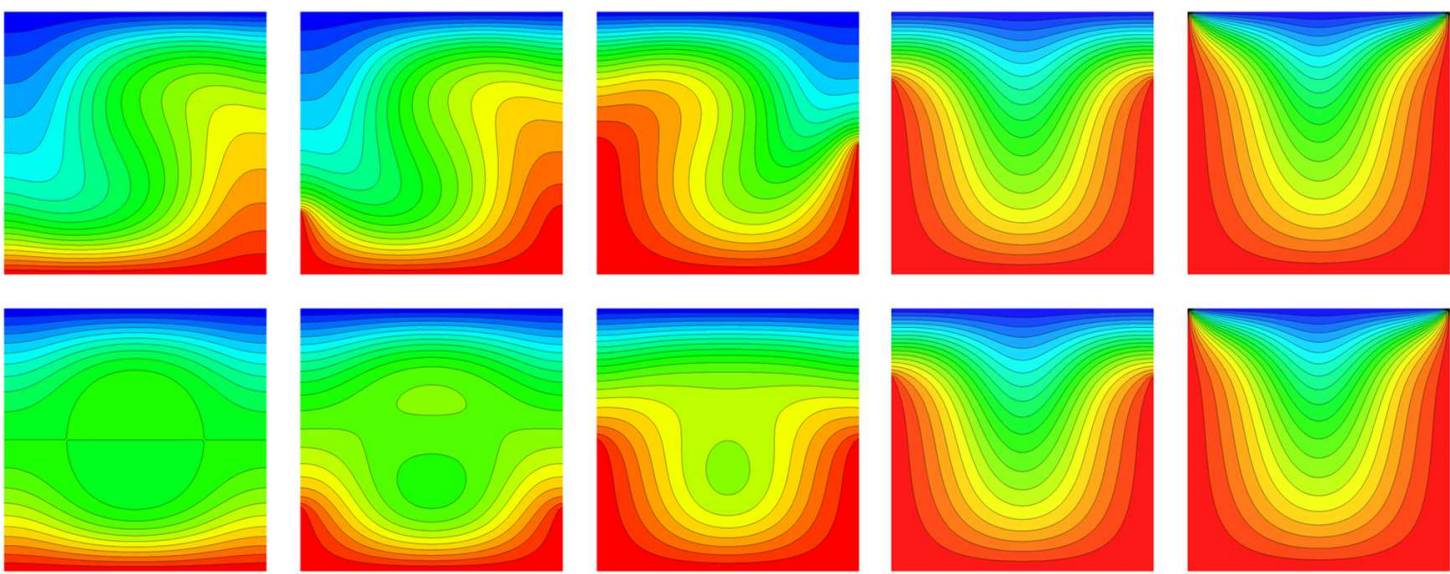

Fig. 3. Steady-state temperature distributions and isotherms at $\mathrm{Ra}=10^{4}, \operatorname{Pr}=6.67$, and $a=1.0$. Top row $\theta=0$ and lower row $\theta=\pi / 2$. From left to right $\gamma=0,0.25,0.5,0.75$, and 1.0 , respectively

The results show that the $\mathrm{Nu}$ number increases as $\gamma$ increases, see Fig. 4. It reaches values in the range of $1.965 \leqslant \mathrm{Nu} \leqslant 7.463$. It should be noticed that near to $\gamma_{c}$, the Nusselt number increases with a different slope.

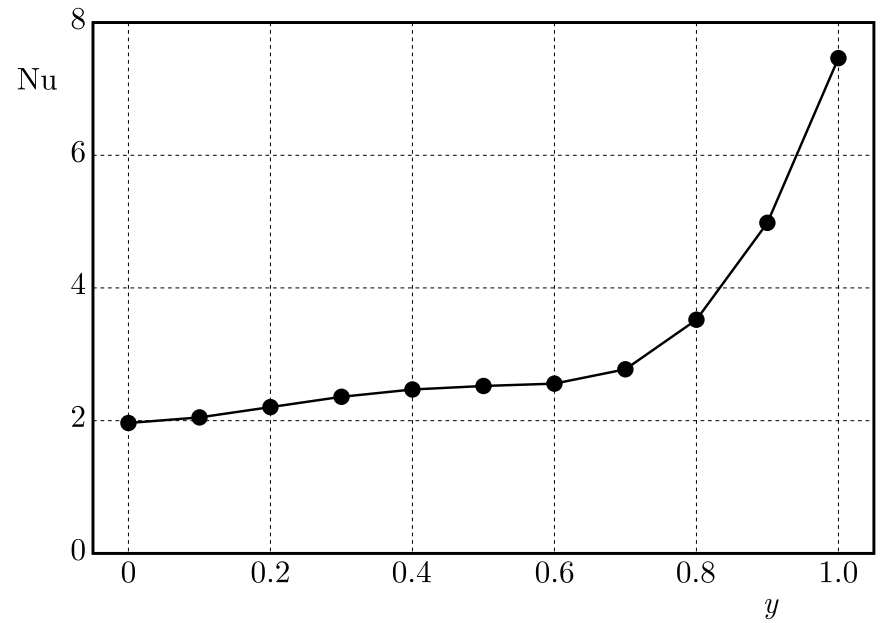

Fig. 4. Nusselt number on the top wall as a function of the heater size, $\operatorname{Ra}=10^{4}, \operatorname{Pr}=6.67$, and $a=1.0$

\subsubsection{Aspect ratio}

Now, we turn our attention to analysis of the effect of the aspect ratio on the flow behavior while keeping the remaining parameters constant: $\operatorname{Ra}=10^{4}, \operatorname{Pr}=6.67$, and $\gamma=0.5$. The results are presented in Fig. 5 for $a=0.5,0.75,1.0$, and 1.25. As in the previous case, symmetry-breaking bifurcations are also observed. For the considered parameters, three solutions are found to be steady non-axisymmetric $C_{1}$. The smallest aspect ratio leads to an axisymmetric configuration $C_{0}$, as shown in Fig. 5. In particular, the flow is axisymmetric for $a<a_{c}=0.669$, calculated by a nonlinear stability analysis (Núñez and Beltrán, 2018), and for $a>a_{c}$ the flow is non-axisymmetric.

\subsubsection{Working fluids}

In previous Sections, we used a high Prandtl number value $\operatorname{Pr}=6.67$. However, in applications like steel production, crystal growth and LMBs, there are low Prandtl number fluid flows 

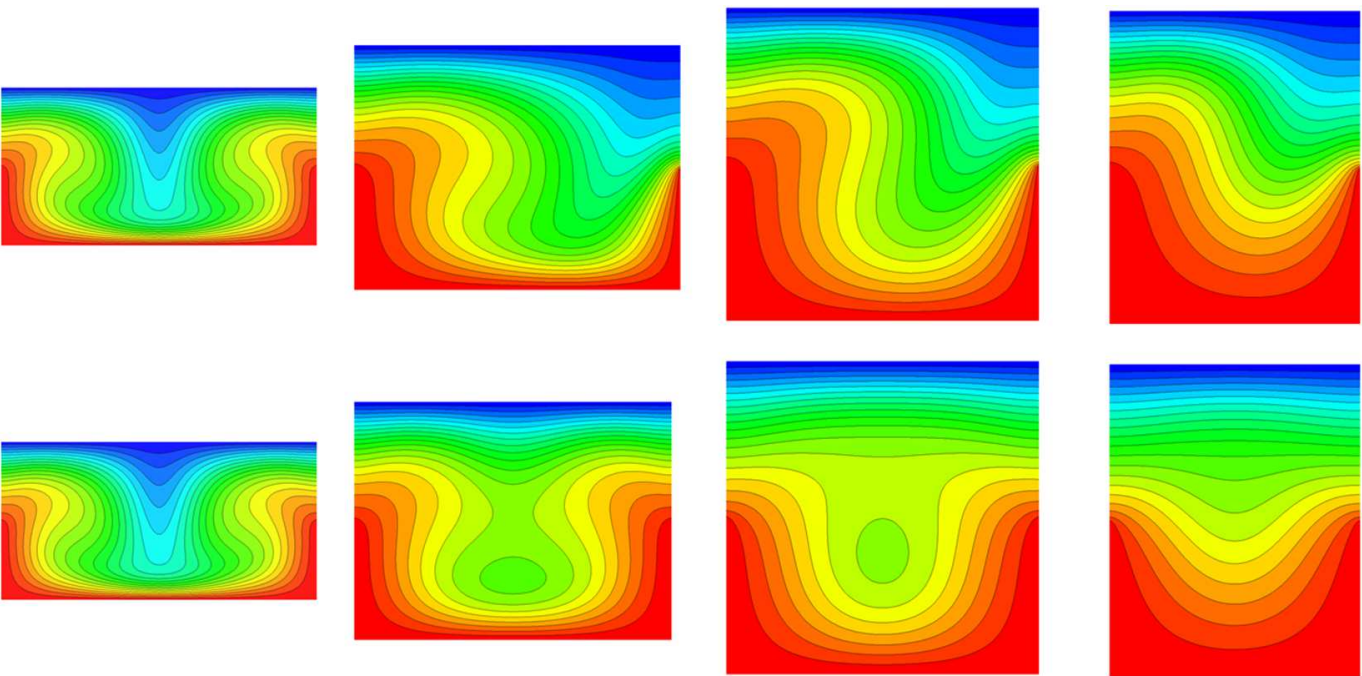

Fig. 5. Temperature distributions and isotherms for $\operatorname{Ra}=10^{4}, \operatorname{Pr}=6.67$, and $\gamma=0.5$. Top row $\theta=0$ and lower row $\theta=\pi / 2$. From left to right $a=0.5,0.75,1.0$, and 1.25 , respectively

involved. Predicting the flow field for low Prandtl number fluids is difficult because the flow is often in the transition range between conduction and convection dominated regimes (Grötzbach, 2013). The flow is also highly nonlinear because the inertial force dominates, and the viscous effects are mainly confined to the very thin boundary layers (Mohamad and Viskanta, 1991).
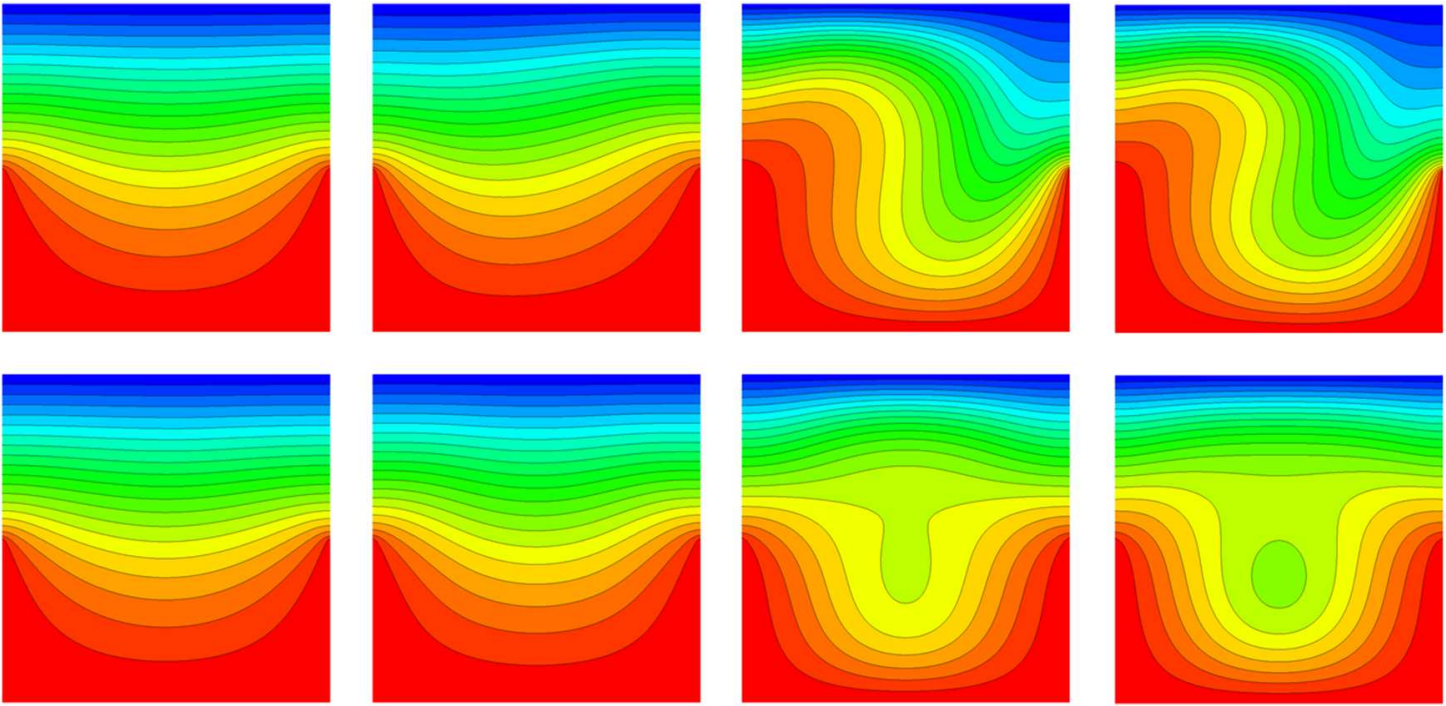

Fig. 6. Temperature distributions and isotherms for $\mathrm{Ra}=10^{4}, a=1.0$, and $\gamma=0.5$. Top row $\theta=0$ and lower row $\theta=\pi / 2$. From left to right $\operatorname{Pr}=0.001,0.01,0.7$, and 6.67 , respectively

Figure 6 shows variations of the flow due to changes in the Prandtl number. These computations have been carried out for $\operatorname{Ra}=10^{4}, a=1.0, \gamma=0.5$, and considering $\operatorname{Pr}=0.001$, $0.01,0.7$, and 6.67. At low values of the Prandtl number, the results show that the temperature field asymptotically approaches an axisymmetric steady-state. At this limit, the Nusselt number reaches a value of around 1.619, see Fig. 7. Horanyi et al. (1999) reported correlations for the calculation of the $\mathrm{Nu}$ number for some liquid metals as a function of the Ra number, for Mercury $(\mathrm{Pr}=0.025)$ they found that $\mathrm{Nu}=0.147 \mathrm{Ra}^{0.257}$. Using the previous correlation, we obtain $\mathrm{Nu}=1.568$, quite similar to the asymptotic value previously mentioned. 


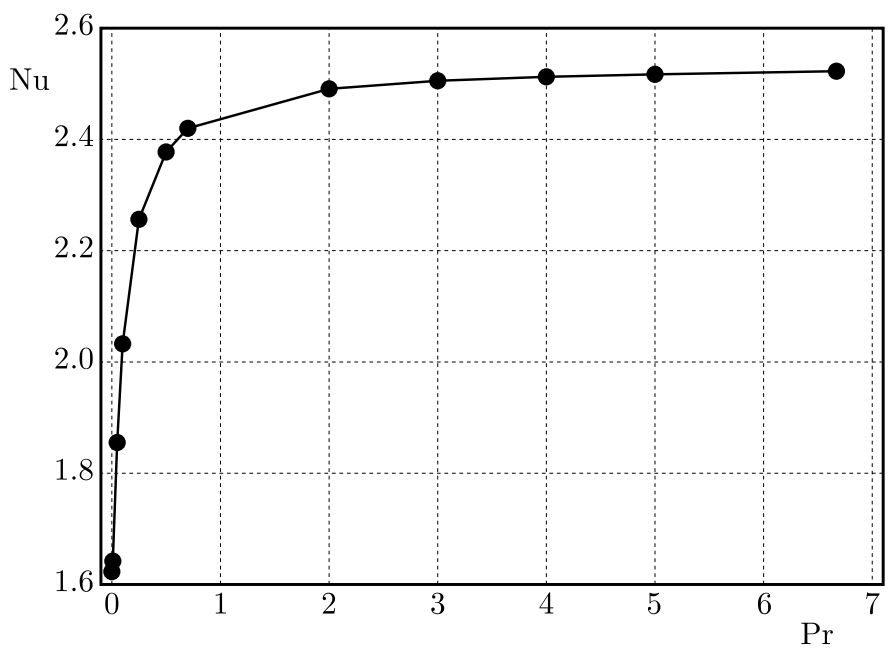

Fig. 7. Nusselt number as a function of the Prandtl number, $\operatorname{Ra}=10^{4}, a=1.0$, and $\gamma=0.5$

\subsection{Flow transitions and bifurcations}

In this Section, we focus our attention on the analysis of nonlinear dynamics of the system and bifurcations through a parametric study, assuming water as the working fluid, $\operatorname{Pr}=6.67$. The Fourier modes solutions are presented in Fig. 8. In these figures, each point corresponds to a numerical solution determined for the flow for $a=1.0$ and ( $\gamma$, Ra) values in the parametric space. A total of 231 calculations for $0.25 \leqslant \gamma \leqslant 0.82$ and $10^{3} \leqslant \mathrm{Ra}<5 \cdot 10^{5}$ have been performed, for 11 aspect ratios and 21 Rayleigh numbers, all equispaced.

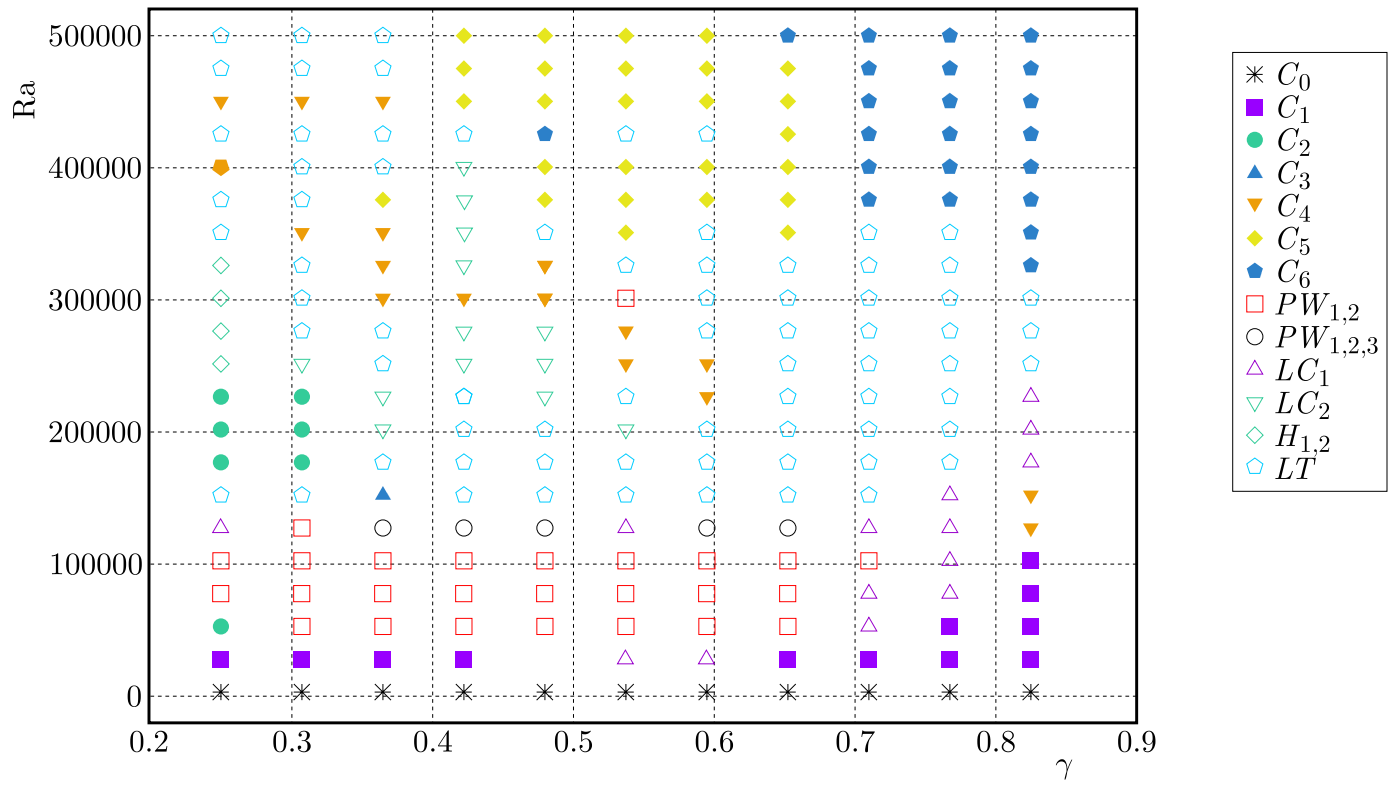

Fig. 8. Fourier modes solutions for $a=1.0, \operatorname{Pr}=6.67$, and $(\gamma, \mathrm{Ra})$ values in the parametric space. Each type of solution is identified with different symbols in the labels of the figure

An aspect ratio of $a=1.0$ was considered to avoid high Fourier mode solutions. Moderate Rayleigh numbers were considered because at higher values the flow leads to a strong modes interaction, and the influence of one particular mode might be not identified. Extreme values of the heater size $(\gamma<0.25$ and $\gamma>0.82)$ are not considered in order to avoid thin thermal boundary layers effects. 
For the smallest value of $\mathrm{Ra}=10^{3}$, convective motions are very slow, and the flow has a steady axisymmetric solution $C_{0}$. Major transitions are observed when increasing the Ra number. Even though the system starts with an entirely symmetrical solution, any small perturbation in the initial conditions will evolve into a non-axisymmetric solution. This situation is referred to as symmetry-broken solutions, which means that the solution has less symmetry than the governing equations. The main pattern observed when the axisymmetric flow is broken corresponds to standing waves $C_{m}$, where the azimuthal wavenumber $m$ depends on $\gamma$ and Ra values. Over the entire region, the numerical solution has a large transient state (LT), which reflects that several solutions can coexist. There are some well-defined regions in the parametric space corresponding to particular modes. Some solutions (like $C_{3}$ and $P W_{1,2}$ modes close to the middle of the map) look isolated, however, they are unstable mode solutions. No attempt was made to show multiple states at the same points in the parameter space that were found using other initial conditions. Other types of flow dynamics that were found are limit cycles $L C_{1}$ and $L C_{2}$, and a double Fourier mode solution $H_{1,2}$. Several authors (Gutierrez-Castillo and Lopez, 2017) identified that saddle-node and Hopf bifurcations were the mechanisms that generate such dynamics in the system, resulting in different coexisting branches of three-dimensional solutions.

(a)

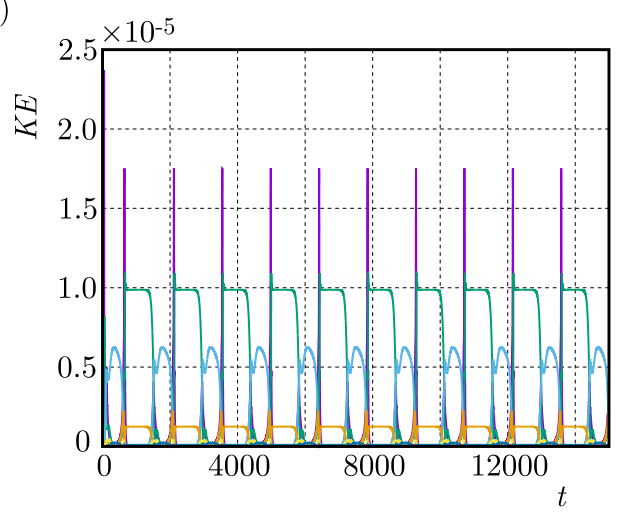

(c)

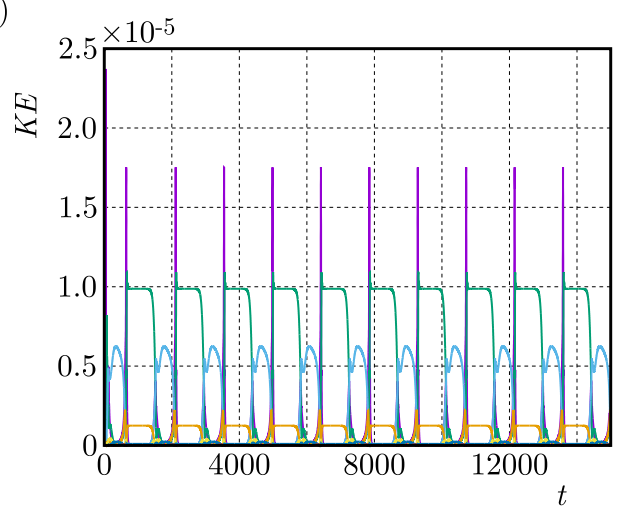

(b)

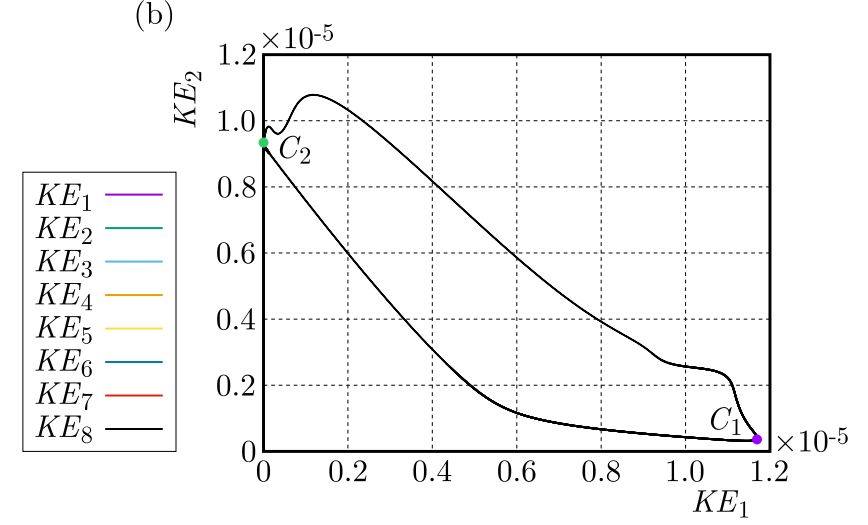

(d)

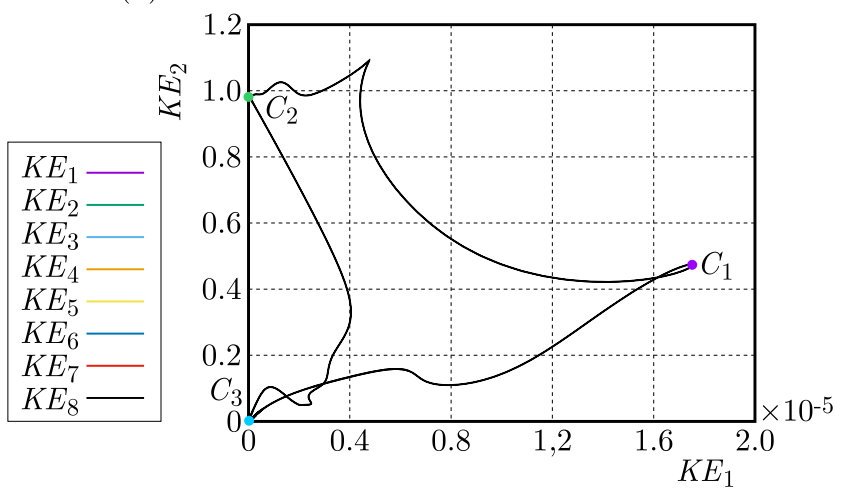

Fig. 9. (a) and (c) Kinetic energies $K E$ as a function of time, obtaining pulsating waves $C_{1}: C_{2}$ and $C_{1}: C_{2}: C_{3}$. (b) and (d) Phase portrait, of $K E_{1}$ vs. $K E_{2}$

For $\gamma=0.5$ and $\mathrm{Ra}=10^{5}$, the flow has two switching mode solutions $C_{1}$ and $C_{2}$, such that the oscillatory behavior can be distinguished when the flow kinetic energy is evaluated, see Fig. 9a. This case is named as the pulsating wave $P W_{1,2}$, where the subindex refers to the involved Fourier modes. The trajectory in the phase portrait for $P W_{1,2}$ closes on itself, it is nearly an elongated ellipse which indicates that the solution switches only from $C_{1}$ to $C_{2}$, see Fig. 9b. For $\mathrm{Ra}=1.272 \cdot 10^{5}$, a switching mode solution $C_{1}, C_{2}$ and $C_{3}$ is observed. It corresponds to a pulsating wave $P W_{1,2,3}$, see Fig. 9c. The kinetic energy in the phase portrait for $P W_{1,2,3}$ is zero in $C_{3}$, see Fig. 9d. The period of the inertial oscillations is found to decrease with Ra as 
can be seen in Fig. 10. At the onset of $P W$, the flow has a very large period, up to 25 periods were calculated for the solution.

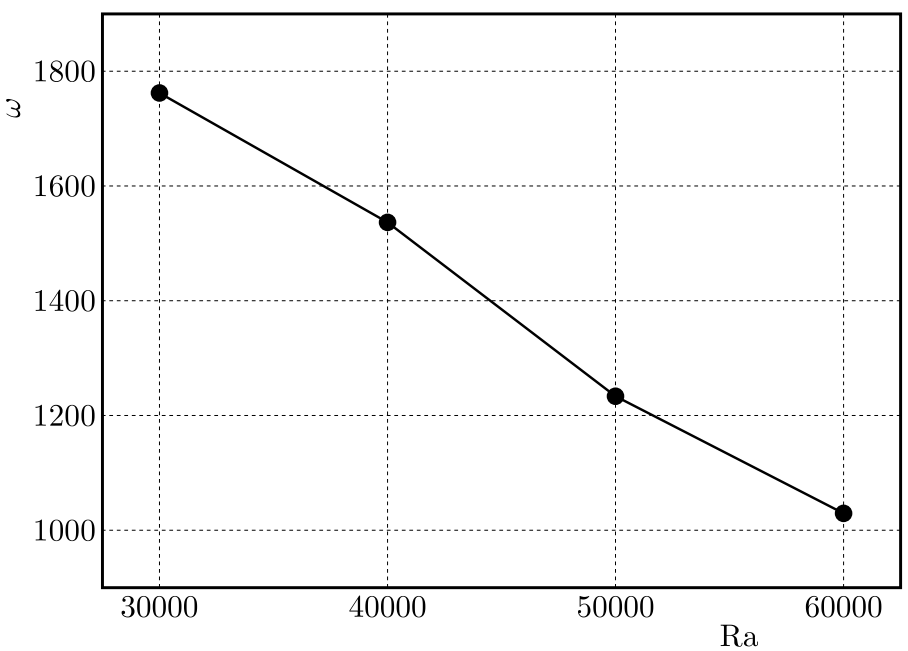

Fig. 10. Precession frequency vs. Rayleigh number for $P W_{1,2}$ solutions

These solutions disappear when other solutions $C_{4}, C_{5}$, and $C_{6}$ take place. The critical Rayleigh number can be estimated through a linear fit when the precession frequency tends to zero, $\omega=2516.04-0.0250112 \mathrm{Ra}$, and it is found that $\mathrm{Ra}_{\omega}=100.596$.

Finally, $C_{1}$ to $C_{6}$ modes can be seen in Fig. 11, and they correspond to steady-state solutions.

$C_{1}$

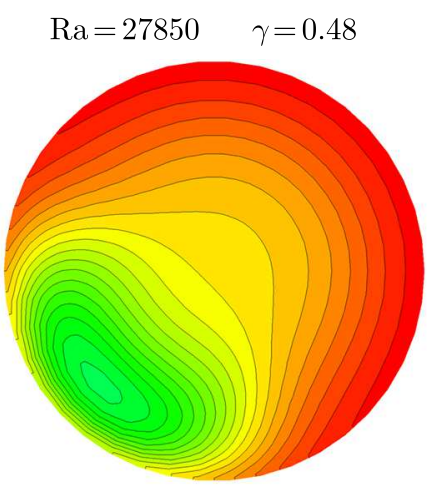

$C_{4}$

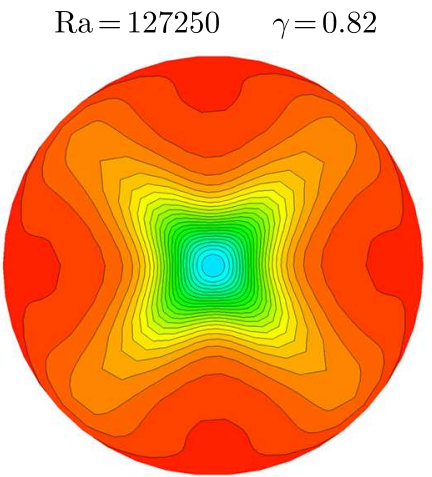

$C_{2}$

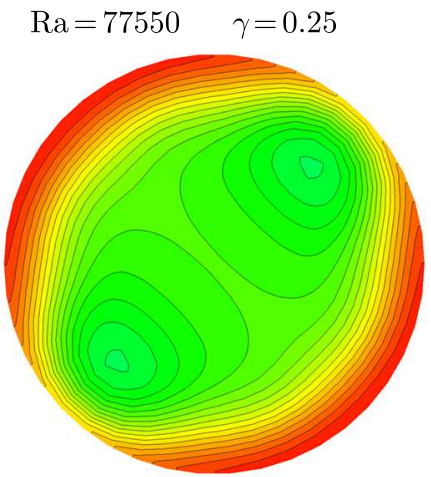

$C_{5}$

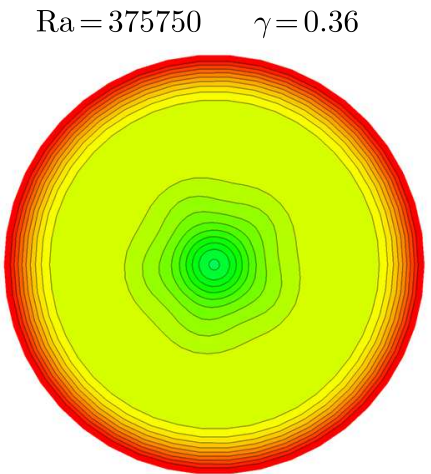

$C_{3}$

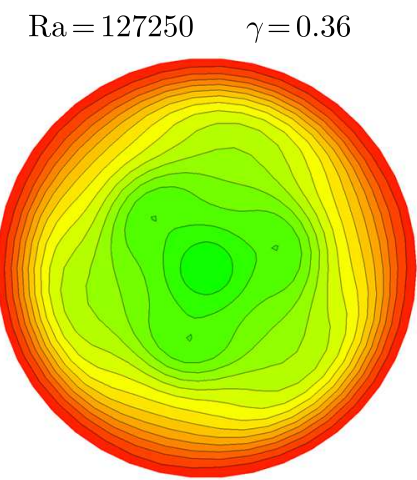

$C_{6}$

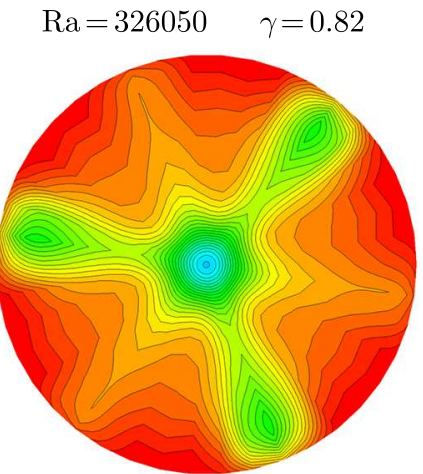

Fig. 11. Fourier modes solutions $C_{1}$ to $C_{6}$. Isothermal contours are presented in the horizontal plane at $z=\gamma$ 


\section{Conclusions}

Natural convection in a cylindrical enclosure with a partially heated lateral wall was numerically studied by using a hybrid Fourier-finite volume code. This code was validated with experimental PIV results reported in the literature for $\operatorname{Pr}=6.67$ (water); whereas, for low Pr numbers, the asymptotic value for the numeric evaluation of the $\mathrm{Nu}$ number is similar to the one obtained from a correlation for mercury $(\operatorname{Pr}=0.025)$. In particular, for $\mathrm{Ra}=10^{4}$, the effect on the heat transfer of parameters like heater size (in the range of $0 \leqslant \gamma \leqslant 1.0$ ), aspect ratio (in the range of $0.5 \leqslant a \leqslant 1.0$ ), and $\operatorname{Pr}$ number (in the range of $0.001 \leqslant \operatorname{Pr} \leqslant 6.67$ ) were detailed analyzed. The following can be concluded for the steady-state temperature distributions: when the heater size was increased, the solutions went from non-axisymmetric to axisymmetric ones; on the other hand, for the increasing of either the aspect ratio or the Pr number, inverse behavior was observed.

The second part of the results was focused on the analysis of non-steady solutions when the Ra number was varied in the range of $10^{3} \leqslant \mathrm{Ra} \leqslant 5 \cdot 10^{5}$. The whole set of solutions in the parametric space $(\gamma, \mathrm{Ra})$ was calculated. These results allowed us to know what was the dominant dynamic according to the conditions of the system. A variety of flow solutions with many spatiotemporal symmetries were found, for instance: standing waves, pulsating waves and limit cycles. More complex solutions were also found by the interaction of two or more Fourier modes. The obtained thermal patterns in the horizontal planes exhibited different pole structures.

For a fixed value of the heater size, Fourier modes appear progressively and grow from zero to six as the Rayleigh number is increased. This study provides basic research on the numerical simulation of natural convection with partial lateral heating, and it exhibits the rich dynamic behavior of the flow.

\section{Acknowledgements}

The authors would like to acknowledge UNAM-DGAPA-PAPIIT Project IA102017 and SENER-CONACYT 2014-246911 Clúster de Biocombustibles Sólidos para la Generación Térmica y Eléctrica for the financial support. Computations were performed at the Laboratory for Design, Modeling, and Simulations (LDMS) at the Morelia Unit of the IIM-UNAM.

\section{References}

1. Al-Rashed A., Kolsi L., Hussein A.K., Hassen W., Aichouni M., Borjini M.n, 2017, Numerical study of three-dimensional natural convection and entropy generation in a cubical cavity with partially active vertical walls, Case Studies in Thermal Engineering, 10, 100-110

2. Beltrán A., 2017, MHD natural convection flow in a liquid metal electrode, Applied Thermal Engineering, 114, 1203-1212

3. Ben-Cheikh N., Campo A., Ouertatani N., Lili T., 2010, Three-dimensional study of heat and fluid flow of air and dielectric liquids filling containers partially heated from below and entirely cooled from above, International Communications in Heat and Mass Transfer, 37, 5, 449-456

4. Bennacer R., El Ganaoui M., Leonardi E., 2006, Symmetry breaking of melt flow typically encountered in a Bridgman configuration heated from below, Applied Mathematical Modelling, 30, $11,1249-1261$

5. Cianfrini C., Corcione M., Habib E., Quintino A., 2013, Convective transport in rectangular cavities partially heated at the bottom and cooled at one side, Journal of Thermal Science, 22, 1, $55-63$

6. Crawford J.D., Knobloch E., 1991, Symmetry and symmetry-breaking bifurcations in fluid dynamics, Annual Review of Fluid Mechanics, 23, 341-387 
7. Erenburg V., Gelfgat A.Yu., Kit E., Bar-Yoseph P.Z., Solan A., 2003, Multiple states, stability and bifurcations of natural convection in a rectangular cavity with partially heated vertical walls, Journal of Fluid Mechanics, 492, 63-89

8. Gelfgat A.Y., 2017, Time-dependent modeling of oscillatory instability of three-dimensional natural convection of air in a laterally heated cubic box, Theoretical and Computational Fluid Dynamics, 31, 4, 447-469

9. GrötzBach G., 2013, Challenges in low-Prandtl number heat transfer simulation and modelling, Nuclear Engineering and Design, 264, 41-55

10. Guestal M., Kadja M., Hoang M.T., 2018, Study of heat transfer by natural convection of nanofluids in a partially heated cylindrical enclosure, Case Studies in Thermal Engineering, 11, $135-144$

11. Gutierrez-Castillo P., Lopez J.M., 2017, Nonlinear mode interactions in a counter-rotating split-cylinder flow. Journal of Fluid Mechanics, 816, 719-745

12. Hasnaoui M., Bilgen E., Vasseur P., 1992, Natural convection heat transfer in rectangular cavities partially heated from below, Journal of Thermophysics and Heat Transfer, 6, 2, 255-264

13. Horanyi S., Krebs L., Müller U., 1999, Turbulent Rayleigh-Bénard convection in low Prandtl-number fluids, International Journal of Heat and Mass Transfer, 42, 21, 3983-4003

14. Kelley D.H., Sadoway D.R., 2014, Mixing in a liquid metal electrode, Physics of Fluids, 26, 5,057102

15. LApPA M., 2005, Review: Thermal convection and related instabilities in models of crystal growth from the melt on Earth and in microgravity: Past history and current status, Crystal Research and Technology, 40, 6, 531-549

16. Lappa M., 2010, Thermal Convection, Patterns, Evolution and Stability, Wiley

17. Lopez J.M., Marques F., 2008, Centrifugal effects in rotating convection: nonlinear dynamics, Journal of Fluid Mechanics, 628, 269

18. Mohamad A.A., Viskanta R., 1991, Transient natural convection of low-Prandtl-number fluids in a differentially heated cavity, International Journal for Numerical Methods in Fluids, 13, 1, $61-81$

19. Nam P.-O., Yi K.-W., 2010, Simulation of the thermal fluctuation according to the melt height in a CZ growth system, Journal of Crystal Growth, 312, 8, 1453-1457

20. Nascimento G., Lugarini A., Germer E.M., Franco A.T., 2019, A simple homogeneous numerical solution for nanofluid natural convection in an enclosure with disconnected and conducting solid blocks, Journal of the Brazilian Society of Mechanical Sciences and Engineering, 41, 6, 262

21. Nithyadevi N., Kandaswamy P., Lee J., 2007, Natural convection in a rectangular cavity with partially active side walls, International Journal of Heat and Mass Transfer, 50, 23-24, 4688-4697

22. NúÑez J., Beltrán A., 2018, On the onset of natural convection in a partially cooled cylinder, Heat Transfer Research, 49, 8, 773-786

23. Núñez J., López-Caballero M., Ramos E., Hernández-Cruz G., Vargas M., Cuevas S., 2012a, Verification and experimental validation of a numerical simulation of natural convection in a slender cylinder, International Journal of Heat and Fluid Flow, 38, 118-125

24. NúÑez J., Ramos E., López J.M., 2012b, A mixed Fourier-Galerkin finite-volume method to solve the fluid dynamics equations in cylindrical geometries, Fluid Dynamics Research, 44, 3, 031414

25. Padilla E.L.M., Lourenço M.A.S., Silveira-Neto A., 2013, Natural convection inside cubical cavities: numerical solutions with two boundary conditions, Journal of the Brazilian Society of Mechanical Sciences and Engineering, 35, 3, 275-283 
26. Patankar S.V., Spalding D.B., 1972, A calculation procedure for heat, mass and momentum transfer in three-dimensional parabolic flows, International Journal of Heat and Mass Transfer, 15, 10, 1787-1806

27. Ramírez G., Núñez J., Hernández-Cruz G., Ramos E., 2020, Natural convective three-dimensional flow structure in a cylindrical container, International Communications in Heat and Mass Transfer, 116, 104616

28. Shen Y., Zikanov O., 2016, Thermal convection in a liquid metal battery, Theoretical and Computational Fluid Dynamics, 30, 4, 275-294

29. Son S.S., Yi K.W., 2005, Experimental study on the effect of crystal and crucible rotations on the thermal and velocity field in a low Prandtl number melt in a large crucible, Journal of Crystal Growth, 275, 1-2

30. Varol Y., Oztop H.F., Koca A., Ozgen F., 2009, Natural convection and fluid flow in inclined enclosure with a corner heater, Applied Thermal Engineering, 29, 2-3, 340-350

Manuscript received May 25, 2021; accepted for print July 5, 2021 